\title{
Correlation between fluxgate and SQUID magnetometer data sets for geomagnetic storms
}

\author{
Thabang Matladi ${ }^{1,2, a}$, Elda Saunderson ${ }^{1, b}$, Daniel Gouws ${ }^{1}$, Temwani-Joshua Phiri ${ }^{1,2}$, \\ Christian Kwisanga ${ }^{2}$ and Coenrad Fourie ${ }^{2}$ \\ ${ }^{1}$ SANSA Space Science, Hospital Street, Hermanus, 7200 Western Cape, South Africa \\ ${ }^{2}$ Dept. E\&E Engineering, Stellenbosch University, Stellenbosch 7600, South Africa
}

\begin{abstract}
There has always been a need to monitor the near Earth's magnetic field, as this monitoring provides understanding and possible predictions of Space Weather events such as geomagnetic storms. Conventional magnetometers such as fluxgates have been used for decades for Space Weather research. The use of highly sensitive magnetometers such as Superconducting QUantum Interference Devices (SQUIDs), promise to give more insight into Space Weather. SQUIDs are relatively recent types of magnetometers that exploit the superconductive effects of flux quantization and Josephson tunneling to measure magnetic flux. SQUIDs have a very broad bandwidth compared to most conventional magnetometers and can measure magnetic flux as low as a few femtotesla. Since SQUIDs have never been used in Space Weather research, unshielded, it is necessary to investigate if they can be reliable Space Weather instruments. The validation is performed by comparing the frequency content of the SQUID and fluxgate magnetometers, as reported by Phiri [1].
\end{abstract}

\section{Introduction}

Superconducting Quantum Interference Device (SQUID) magnetometers use the Josephson Effect joint with flux quantization to measure relative changes in the applied magnetic flux [2]. They are highly sensitive and have a very broad bandwidth: they can measure magnetic fields as low as a few femtotesla, with a bandwidth from quasi-DC to a few $\mathrm{GHz}$ [3]. Due to their sensitivity and broad bandwidth, SQUIDs may be used in Space Weather research to compliment already existing Space Weather instruments, and also give more insight where some magnetometers have limitations. They have low noise levels, thus enabling them to resolve faint signals that most conventional magnetometers are incapable of resolving.

In Space Weather, magnetometers such as fluxgates are generally used to monitor the near-Earth magnetic field. The monitoring of the geomagnetic field is equally important to scientists and to society as a whole. Space Weather events such as geomagnetic storms are capable of disrupting or even destroying our technological systems, both in space and on the ground. It is through monitoring the

\footnotetext{
a e-mail: tkmatladi@gmail.com

b e-mail: esaunderson@sansa.org.za
}

This is an Open Access article distributed under the terms of the Creative Commons Attribution License 4.0, which permits unrestricted use, distribution, and reproduction in any medium, provided the original work is properly cited. 
Earth's magnetic field that we learn more about these Space Weather events and develop models to predict such events. Research has shown that, using highly sensitive magnetometers such as SQUIDs, electromagnetic signals emitted prior to an earthquake can be detected $[4,5]$. Thus using SQUIDs for Space Weather research may also enable the observation of possible earthquake precursors.

SQUID magnetometers are used in shielded environments in many fields such as medical studies, as well as unshielded operations such as geophysical exploration. However, it is necessary to investigate whether SQUIDs could be reliable Space Weather instruments when operated unshielded. The validation may be done by comparing SQUIDs with other conventional magnetometers that have been in use for Space Weather research and have been proven to be reliable, such as fluxgate magnetometers. In this paper, we present a correlative study that was done at the South African National Space Agency (SANSA), Directorate: Space Science, Hermanus, South Africa, between SQUID and fluxgate magnetometer data sets for geomagnetic storms occurring in the year 2013. The aim of the study is to validate the use of SQUIDs for Space Weather research, by comparing the frequency content of the SQUID data with that of the fluxgate data.

This study is based on the scientific study that was conducted at SANSA Space Science during 2012 [1]. In that study, the frequency content (matching spectral components, or peaks) of the SQUID data was compared to the frequency content of the three fluxgate data sets for geomagnetic storms that occurred in the year 2011. The SQUID data was obtained from a Low-T 3 -axis SQUID located in the Low Noise Laboratory (LSBB) in France, with the fluxgate data sets acquired from the three closest magnetic observatories to LSBB. Those three observatories are Chambon la Forêt (CLF, France), Ebro (EBR, Spain) and Fürstenfeldbruck (FUR, Germany), of which are all more than $500 \mathrm{~km}$ from LSBB. It was established that the correlation between the SQUID and fluxgate data sets was, at the very least, $59 \%$, and that the SQUIDs can be used as a reliable Space Weather research tool.

There are significant differences between the previous and the current study. The first study used a Low- $\mathrm{T}_{\mathrm{c}}$ 3-axis ( $\left.x y z\right)$ SQUID located in a partially shielded chamber, while in this study a 2-axis $(x z)$ High- $\mathrm{T}_{\mathrm{c}}$ SQUID is used instead, operated in a completely unshielded environment. The SQUID and fluxgates used in this study are also located within $50 \mathrm{~m}$ from each other; thus will enable us to isolate magnetic signals detected by SQUID and not by the fluxgate, and the correlation is expected to be higher than the correlation obtained in the previous study.

\section{Methods and materials}

The data sets used in this study were acquired from SQUID and fluxgate magnetometers, which are located within $50 \mathrm{~m}$ from each other at SANSA Space Science. The observatory is situated near the Atlantic Ocean and a small industrial area, in the coastal town of Hermanus. The SANSA facility is magnetically clean to magnetic observatory standards and is part of the world-wide network of magnetic observatories: International Real-time Magnetic Observatory Network (INTERMAGNET).

\subsection{Fluxgate data}

The fluxgate data are acquired using a 3-axis FGE fluxgate magnetometer manufactured by the Danish Meteorological Institution in Denmark. The FGE fluxgate monitors the $h d z$ geomagnetic components, with a dynamic measurement range of $3000 \mathrm{nT}$ and noise level of $40 \mathrm{pT}$ at $1 \mathrm{~Hz}$. Since the fluxgate data is compared to the SQUID data, which are $x z$ components, the fluxgate $x$ component has to be derived trigonometrically from $h$ and $d$ using a cosine function. The fluxgate records data every second, with the data sampled every 5 seconds. To meet INTERMAGNET data specifications, a numerical filter is then applied to produce a 1 minute data. The data are available at (http://intermagnet.org/) and also on-site, at SANSA Space Science. 


\subsection{SANSA Space Science SQUID system}

The SANSA SQUID system is a 2-axis High- $\mathrm{T}_{\mathrm{c}}$ device, measuring the $x z$ geomagnetic components. The M2700 SQUID sensors from Star Cryoelectronics, with intrinsic noise levels less than $300 \mathrm{fT} / \mathrm{Hz}^{1 / 2}$ above $10 \mathrm{~Hz}$, have a dynamic range of $450 \mathrm{nT}$. The sensors operate at a temperature of $77 \mathrm{~K}$ in liquid nitrogen contained in a non-magnetic dewar, which requires monthly refilling. The output of the sensors are recorded using a National Instruments data acquisition unit (NI-DAQ USB6281), sampled at $125 \mathrm{~Hz}$. Moreover, the DAQ could be sampled up to $500 \mathrm{kHz}$ for high frequency measurements [6]. The data acquisition system limits the SQUID data to a resolution of about $5 \mathrm{pT}$. The resolution may be improved in the future by using a 24-bit DAQ. SQUID data are accessible via (http://geomagnet.ee.sun.ac.za/), or on-site.

The SQUID sensors are mounted on a non-magnetic rig which is used to orientate the sensors in the $x-y$ plane and to lift the sensors in and out of the dewar during liquid nitrogen refills. To minimize vibrations due to local disturbances, the dewar and the rig are both placed on concrete pillars which are built on compressed sand and decoupled from each other. Moreover, the floor and foundations of the SQUID hut (a non-magnetic hut that houses the SQUID system) are also decoupled from the SQUID's dewar and rig pillars. The SQUIDs operate in an unshielded environment, in a local geomagnetic field of $23.6 \mu \mathrm{T}$ in the vertical $(z)$ direction and $9.6 \mu \mathrm{T}$ in the horizontal $(x)$ direction.

\section{Results}

The frequency content of the SQUID data was correlated to the frequency content of the fluxgate data for geomagnetic storms occurring during the year 2013. Note that the correlation here means investigating if the frequency peaks present in fluxgate data are also observed in the SQUID data. The storms were selected based on the Dst index, see (http://wdc.kugi.kyoto-u.ac.jp/dst_realtime/) for realtime Dst index plots. For each storm the main phase of the storm was studied.

The SQUID and fluxgate data sets were correlated from the $1 \mathrm{mHz}$ to $8 \mathrm{mHz}$ frequency range. The lower limit of this frequency range was due to excessive 1/f noise on SQUID data below $1 \mathrm{mHz}$, while the $8 \mathrm{mHz}$ limit is due to the Nyquist frequency of the fluxgate data. Many of the geophysical phenomena, such as geomagnetic storms, lighting and ground motions (earthquakes), are known to produce geomagnetic pulsations with some frequency peaks within the $1-8 \mathrm{mHz}$ frequency range [7]. Frequency peaks that were within 5\% from each other were considered to be coinciding.

\subsection{Storm analysis}

A total of six storms were studied, occurring during May, July and October, 2013. The storm that took place on the $1^{\text {st }}$ of May is Storm 1, Storm 2 is took place on the $25^{\text {th }}$ of May, Storm 3 occurred on the $6^{\text {th }}$ of July, with Storm 4 on the $10^{\text {th }}$ to $11^{\text {th }}$ of July, while Storm 5 took place on the $14^{\text {th }}$ of July and the last storm, which occurred on the $2^{\text {nd }}$ of October, referred to as Storm 6. Due to their Kp and Dst indices, Storms 2 and 4 were classified as minor storms, while Storms 1, 3 and 5 were classified as moderate and Storm 6 as intense. The frequency content of the SQUID and fluxgate data sets were obtained by computing an Amplitude Spectral Density (ASD) on a full day's data for each storm.

For Storm 1, 93.11\% of the peaks found in the fluxgate data were also present in the SQUID data. About $90 \%$ of the SQUID peaks that coincided with fluxgate peaks were within $0.5 \%$ of the fluxgate peaks. For Storm 2, shown in Fig. 1, the SQUID data correlated 100\% with the fluxgate data, with over $92 \%$ of the SQUID peaks lying within $0.5 \%$ of the fluxgate peaks. The SQUID data correlated $84.78 \%$ with the fluxgate data for Storm 3, with about $66 \%$ being within $0.5 \%$ of the fluxgate peaks. For Storm 4 the correlation was $89.01 \%$, with over $75 \%$ of the SQUID peaks lying within $0.5 \%$ of fluxgate peaks. The SQUID correlated $90.95 \%$ and $84.03 \%$ for Storms 5 and 6 respectively. Over $75 \%$ of the SQUID 


\section{E3S Web of Conferences}

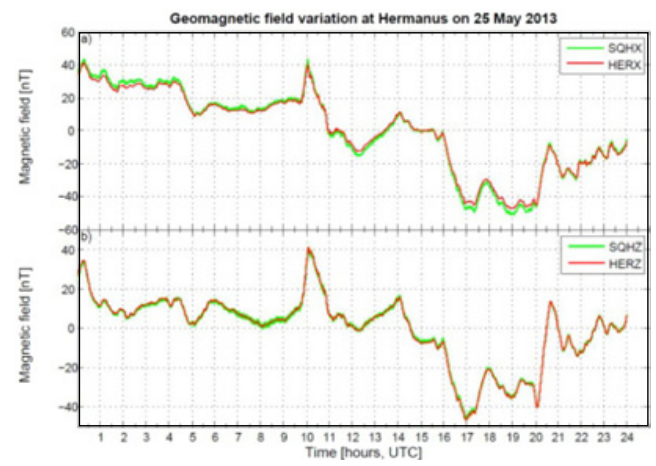

Figure 1. Time domain comparison: Storm no 2.

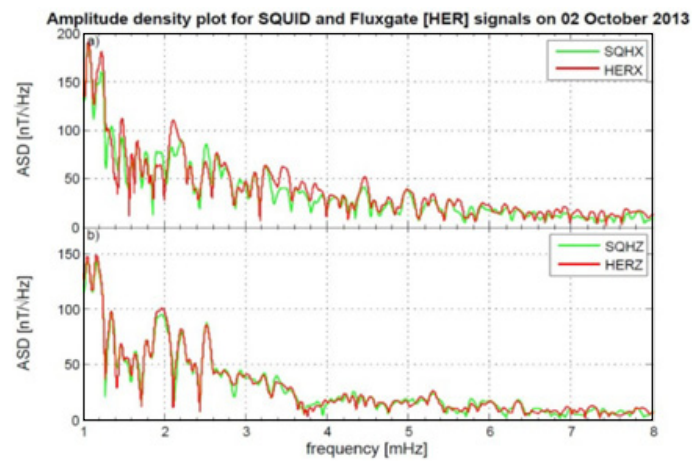

Figure 2. ASD comparison: Storm no 6.

peaks lay within $0.5 \%$ of the fluxgate peaks for Storm 5, while for Storm 6 it was over 85\%, as shown in Fig. 2.

\section{Conclusion}

The correlation was performed between SQUID and fluxgate data sets for geomagnetic storms that occurred during 2013. The main objective of the study was to investigate whether a SQUID magnetometer operated in a completely unshielded geomagnetic field will perform as good as the fluxgate magnetometer, which have been used for decades in Space Weather research and is known to be reliable. The comparison between the SQUID and fluxgate data sets showed good agreement with the correlation, at the very best, as high as $100 \%$ and $84.03 \%$ at the very least. The SQUID data correlated $90.95 \%$ with the fluxgate, overall. Thus the SQUID magnetometer could be considered to perform as good as the fluxgate. The high correlation achieved in the study was to be expected since the SQUID and fluxgate magnetometers are located with $50 \mathrm{~m}$ from each other.

The proximity of the SQUID to the fluxgate also helped to isolate the signals that can be detected by the SQUID but not the fluxgate. For instance, for Storms 3 and 4, there were some prominent peaks on the SQUID $x$ component data which were absent in the fluxgate data. In both cases, the peaks appeared within the $4-6 \mathrm{mHz}$ frequency range. These disturbances are probably due to local sources, i.e., sources that only affect the SQUID but not the fluxgate. 
The results obtained in this study further supports the use of the SANSA SQUID in the proposed long-term Advanced Ultrasensitive real-Time Upgraded Magnetic sensor or Network (AUTUMN) project for studying earthquakes and Space Weather electromagnetic signals. Under the right conditions the electromagnetic signals emitted before an earthquake could be detected using a SQUID magnetometer, thus this network could serve as an early warning system for natural hazards such as earthquakes. The SANSA SQUID magnetometer could be used in the future to study geophysical phenomena, for example, the effect of lightning, rain or strong winds on geomagnetic data. Since the SQUID is also located near the ocean, it can further be used to study the effect of tidal motions on geomagnetic data.

Schumann resonances are natural electromagnetic standing waves in the Earth-Ionosphere waveguide, created by thunderstorm activity, with the peak resonances located at $8,14,20,26,33 \mathrm{~Hz}$, etc. We have observed Schumann resonances in the $12-14 \mathrm{~Hz}$ range in the SANSA SQUID data, and more research is currently underway on this topic at SANSA Space Science.

\section{References}

[1] T.-J. Phiri, Masters Thesis - Stellenbosch University (2012).

[2] R. Cantor, D. Koelle The SQUID Handbook 1st ed vol 1 (2004).

[3] D. Drung, J. Physica C 368, 134-140 (2002).

[4] G. Waysand, P. Barroy, R. Blancon, S. Gaffet, C. Guilpin, J. Marfaing, E. Pozzo di Borgo, M. Pyée, M. Auguste, D. Boyer, A. Cavaillou, Eur. Phys. J. Appl. Phys., 47-1 (2009).

[5] G. Waysand, J. Marfaing, E. Pozzo di Borgo, R. Blancon, M. Pyée, M. Yedlin, P. Barroy, M. Auguste, D. Boyer, A. Cavaillou, J. Poupeney, C. Sudre, C.R. Physique, 12, 192-202 (2011).

[6] L.J. Janse van Vuuren, A. Kilian, T.J. Phiri, C.J. Fourie, E. Pozzo di Borgo, P. Febvre, E.F. Saunderson, E.T. Lochner and D.J. Gouws, Proc. IEEE AFRICON 2013, 908-912 (2013).

[7] McPherron, Surv. Geophys., 26 545-592 (2005). 